\title{
Errores de refracción en niños de 6 a 8 años y factores asociados. Estudio transversal analítico
}

\section{Refractive errors in children aged 6 to 8 years and associated factors. Cross-sectional analytical study}

\author{
Carlos J. Avendaño-Vásquez ${ }^{*}$, Jeimy N. Gutiérrez-Rodríguez², Javier A. Rodríguez-Hilarión ${ }^{1}$ y \\ Magda S. Ortiz-Clavijo ${ }^{3}$ \\ ${ }^{1}$ Facultad de Enfermería; ${ }^{2}$ Facultad de Optometría; ${ }^{3}$ Facultad de Psicología. Universidad Antonio Nariño, Bogotá, Colombia
}

\begin{abstract}
Resumen
Los errores de refracción (ER) han sido motivo de interés mundial por ser considerados el problema ocular más común en todos los grupos de edad. Reconocer su carácter multifactorial es vital para dar respuesta a través de programas de prevención, diagnóstico y tratamiento oportuno. Objetivo: Analizar la asociación de ER con factores sociales, nutricionales, psicológicos y estilos de vida en niños de 6 a 8 años. Método: Estudio transversal analítico realizado a 149 niños de 6 a 8 años. Valoración de optometría bajo cicloplejia, antropometría y cuestionarios de características sociodemográficas, estilos de vida y personalidad fueron aplicados. Resultados: La prevalencia de hipermetropía fue del 33,5\% y de astigmatismo del 18,7\%, con un incremento de este último directamente proporcional a la edad. Niños con hipermetropía mostraron condiciones socialmente menores (52\%). No se hallaron diferencias estadísticamente significativas en relación con el estado nutricional, pero sí con el consumo de alimentos como azúcares y lácteos ( $p=0,030$ y $p=0.017$, respectivamente). Factores de riesgo como exposición a tabaco o consumo de alcohol fueron identificados en el $14.1 \%$ de niños emétropes. Los niños hipermétropes mostraron menores capacidades frente a escalas de seguridad y duda estadísticamente significativas $(p=0.017)$ en comparación con niños emétropes y con astigmatismo. Conclusión: Los niños con hipermetropía demostraron mayores factores de riesgo relacionados con estilos de vida y rasgos de personalidad. La tendencia creciente de astigmatismo en nuestra población de estudio sugiere medidas de intervención precoces para disminuir su impacto en el contexto escolar. Se sugieren estudios longitudinales que contemplen patrones de conducta modificables para hallar significancia estadística.
\end{abstract}

Palabras clave: Errores de refracción. Hiperopía. Astigmatismo. Niño. Estilos de vida saludable. Determinación de la personalidad. Estado nutricional.

\section{Abstract}

Refractive errors (RE) have been of global interest because they are considered the most common ocular problem in all age groups. Recognizing its multifactorial character is vital to respond through prevention, diagnosis and timely treatment programs. Objective: To analyze the correlation between sociodemographic, nutritional, psychological and lifestyle factors with refractive errors in children. Methodology: Analytical cross-sectional study conducted on 149 children aged 6 to 8 years. Optometric assessment under cycloplegia, anthropometry and application of questionnaires regarding sociodemographic

Correspondencia:

*Carlos J. Avendaño-Vásquez

Universidad Antonio Nariño, Facultad de Enfermería

Carrera 3 este \# 47a-15

Fecha de recepción: 19-08-2019

Fecha de aceptación: 06-05-2020

E-mail: cjavasquez@uan.edu.co
Disponible en internet: 01-07-2020 Rev Mex Oftalmol. 2020;94(4):166-177

www.rmo.com.mx 0187-4519/๑ 2020 Sociedad Mexicana de Oftalmología. Publicado por Permanyer. Este es un artículo open access bajo la licencia CC BY-NC-ND (http://creativecommons.org/licenses/by-nc-nd/4.0/). 
characteristics, lifestyles and personality. Results: The prevalence of hyperopia was $33.5 \%$ and of astigmatism $18.7 \%$, with an increase in the latter directly proportional to age. Children with hyperopia showed socially disadvantaged conditions (52\%). No statistically significant differences were observed regarding nutritional status, but there was a correlation with the consumption of foods such as sugars and dairy products ( $p=0.030$ and $p=0.017$, respectively). Risk factors such as exposure to tobacco or alcohol consumption were identified in $14.1 \%$ of emmetropic children. Hyperopic children showed lower capacities regarding statistically significant safety and doubt scales $(p=0.017)$ compared to emmetropic and hyperopic children. Conclusion: Children with hyperopia showed more risk factors related to lifestyle and personality traits. The growing tendency of astigmatism in our study population should drive early intervention measures to reduce its impact on the school context. Longitudinal studies are suggested that include modifiable behavior patterns to evaluate statistical significance.

Key words: Refractive errors. Hyperopia. Astigmatism. Children. Healthy lifestyles. Personality determination. Nutritional status.

\section{Introducción}

Los errores de refracción (ER) han sido motivo de interés a nivel mundial por ser considerados el problema ocular más común en todos los grupos de edad, por su impacto en la pérdida visual y costos en la atención sanitaria, los cuales producen una pérdida anual que asciende a 269 billones de dólares ${ }^{1}$. La etiología de los ER en población infantil ha sido atribuida a algunos factores sociodemográficos como la edad, sexo, raza y nivel educativo; y a estilos de vida, incluyendo aspectos como la actividad física, nutrición, exposición a tóxicos, patrones de sueño y salud general. Por otra parte, también se han realizado estudios ligando los ER y la expresión de los rasgos de personalidad y el estado emocional $^{2-6}$, con el objetivo de analizar la multidimensionalidad de esta condición y dar respuesta a los interrogantes que surgen en la medida en que se modifican los patrones de vida de la población, para la formulación de programas con impacto en la prevalencia e incidencia de los ER, los cuales ya son considerados como un problema de salud pública. En este sentido, realizar un diagnóstico precoz de los ER e intervenir de manera oportuna los factores que inciden en su etiología puede mejorar la salud visual de la población infantil, y es de suma importancia para dar respuesta a los programas dirigidos a disminuir las brechas en la prevención, detección y atención de los $\mathrm{ER}^{1,7,8}$.

Según la Organización Mundial de la Salud (OMS), «un $80 \%$ de las causas de discapacidad visual son prevenibles o curables" ${ }^{9}$, y es de especial prioridad la atención en menores de 15 años, por la posibilidad de implementar intervenciones efectivas y prevenir implicaciones desfavorables en el desarrollo, acceso a la educación, aprendizaje y, por ende, la calidad de vida de los niños ${ }^{10}$. Si bien en Colombia y otros países de Latinoamérica hay bajo reporte de estudios de prevalencia, algunas investigaciones sostienen un incremento de la miopía, con gran variación según la edad, etnia y factores sociodemográficos, y un comportamiento estable en el caso de la hipermetropía y el astigmatismo en los últimos 40 años $^{7,11}$. En este sentido, las investigaciones en torno al comportamiento epidemiológico de los ER están en incremento, pero su estudio, en relación con los factores desencadenantes de tipo social o psicológico, aún son incipientes.

Bajo este contexto, y de acuerdo con la OMS, la salud visual debe «integrarse en marcos más amplios sobre enfermedades no transmisibles y transmisibles" ${ }^{9}$, lo que implica la necesidad de realizar estudios interprofesionales en el marco de la prevención en población infantil, para intervenir desde diferentes enfoques las principales causas o factores desencadenantes, reconociendo la etiología multifactorial de los $E^{12}$ y aportando a las iniciativas de cuidado de la salud en etapas posteriores de la vida y en grupos marginados y vulnerables.

Bajo tales afirmaciones, este estudio se presenta como uno de los primeros en realizar un análisis de los ER en población infantil escolarizada con el objetivo de analizar su relación con estilos de vida y rasgos de personalidad, realizando una aproximación de la prevalencia y su manifestación a partir de condiciones psicosociales de niños y niñas colombianos, como aporte al reconocimiento de los ER desde una visión integral y multidisciplinaria.

\section{Objetivo}

Analizar la asociación de los ER con factores sociales, nutricionales, psicológicos y estilos de vida en niños de 6 a 8 años.

\section{Metodología}

\section{Diseño del estudio y participantes}

Estudio transversal analítico, realizado a 149 niños de 6 a 8 años durante el segundo semestre de 2018, 
de dos sedes de una institución educativa pública, ubicada en la localidad de Ciudad Bolívar de la ciudad de Bogotá, Colombia, caracterizada por estados marginados poblacionales. Se realizó un muestreo aleatorio simple en un universo de 235 niños, tomando como frecuencia anticipada la prevalencia reportada para astigmatismo del $27.2 \%$, el cual es definido como el ER de mayor frecuencia para el 2018 en el continente americano ${ }^{1}$. El estudio aplicó un índice de confianza de $95 \%$ y un error estándar del $5 \%$. Se obtuvo consentimiento informado del cuidador primario y asentimiento informado de los niños. Los criterios de exclusión fueron niños con déficit cognitivo o con enfermedades oftalmológicas previas.

\section{Procedimientos e instrumentos}

El examen refractivo se realizó siguiendo el protocolo de la Red Epidemiológica Iberoamericana en Salud Visual y Ocular, que establece la aplicación de una gota de benoxinato al $0.4 \%$, una de tropicamida al $1 \%$ y una de ciclopentolato al $1 \%$ (con intervalo de aplicación de 1 a 5 minutos entre cada una). Posteriormente, a los 30 minutos, se valoró el diámetro pupilar esperando una dilatación mayor o igual a $6 \mathrm{~mm}$ de diámetro y reacción pupilar negativa a la luz, momento en el cual se determinó la refracción de cada uno de los niños.

El diagnóstico de los ER se realizó de acuerdo con el estudio de error refractivo en niños ${ }^{13}$ y la Guía de práctica clínica para la detección temprana, el diagnóstico, el tratamiento y el seguimiento de los defectos refractivos en menores de 18 años de Colombia ${ }^{10}$.

Para la definición de las ametropías bajo cicloplejia se usó el equivalente esférico $(E E)$, calculado como el valor de la esfera más la mitad del cilindro, y que este último no sea mayor a - 0.50 dioptrías (D), para definir emetropía, hipermetropía, miopía y astigmatismo. La emetropía se definió con el EE entre $-0.25 \mathrm{D}$ y $+1.75 \mathrm{D}$; la hipermetropía con el $\mathrm{EE} \geq+2.00$ $\mathrm{D}$; la miopía con el $\mathrm{EE} \leq-0.50 \mathrm{D}$, y el astigmatismo se definió teniendo en cuenta que el cilindro presentara un valor mayor o igual a la tercera parte de la esfera, positivo o negativa.

La clasificación antropométrica del estado nutricional se realizó siguiendo los indicadores antropométricos, los patrones de referencia y los puntos de corte de los niños, niñas y adolescentes establecidos por el Ministerio de Salud y Protección Social de Colombia, que, a su vez, se basan en las recomendaciones establecidas por la OMS en materia nutricional a nivel mundial ${ }^{14}$.
Para la clasificación antropométrica del estado nutricional de los niños se tuvo en cuenta el indicador y el punto de corte establecido por desviación estándar (DE). Para el indicador talla para la edad (T/E), DE $\geq-1$ (talla adecuada para la edad); DE $\geq-2$ a $<-1$ (riesgo de retraso en talla); $D E<-2$ (talla baja para la edad 0 retraso en talla). Para el indicador índice de masa corporal para la edad (IMC/E), DE > +2 (obesidad); $\mathrm{DE}>+1$ $a \leq+2$ (sobrepeso); $D E \geq-1$ a $\leq+1$ (IMC adecuado para la edad); $D E \geq-2 \mathrm{a}<-1$ (riesgo de delgadez) y $\mathrm{DE}<-2$ (delgadez) $^{14}$.

Para la toma de peso, se utilizó una báscula previamente calibrada con capacidad de $150 \mathrm{~kg}$, mecanismo digital y precisión de $50 \mathrm{~g}$. La báscula se ubicó en una superficie plana y la medida se tomó con el niño en bipedestación con la menor ropa posible. El peso se tomó en dos momentos para validar la sensibilidad de la báscula. Para la toma de la talla, se utilizó un tallímetro portátil con capacidad de $2 \mathrm{~m}$ y con sensibilidad de $1 \mathrm{~mm}$, el cual fue fijado con tornillos a la pared. La medida se tomó en dos oportunidades y se repitió en los casos con variabilidad mayor a 0.5 centímetros $(\mathrm{cm})^{14}$.

Los datos sociodemográficos se obtuvieron por medio de una encuesta de las condiciones sociales y económicas de la población, que contenía preguntas sobre consumo de alimentos y hábitos de sueño y descanso. Para identificar los estilos de vida saludables, se aplicó el instrumento FANTÁSTICO adaptado a la población colombiana por Betancurth, et al. en $2015^{15}$. Este instrumento está compuesto por 30 preguntas que integran 10 dimensiones: $F$ : familiares y amigos, A: actividad física y social, N: nutrición, T: toxicidad A: alcohol, S: sueño y estrés, T: tipo de personalidad y actividades, I: imagen interior, C: control de la salud y sexualidad y, finalmente, O: orden. Cada dimensión tiene opciones de respuesta con valor numérico de 0 a 2, y se califican por medio de una escala tipo Likert. La puntuación del instrumento varía de 0 a 120 puntos; a mayor puntaje de la dimensión, la calificación es más positiva frente a los hábitos y costumbres relacionados con el estilo saludable de vida $^{15}$.

El perfil psicológico de los niños se evaluó mediante el Cuestionario de personalidad para niños ESPQ, elaborado por Coan y Cattell en 1966 y adaptado al español por TEA Ediciones en 1998. El cuestionario está dirigido a niños entre 6 y 8 años que se ubican escolarmente en los cursos de primero, segundo y tercero de educación básica primaria. El cuestionario consta de 160 preguntas, que miden 13 factores de primer orden de personalidad, que son: factor A (reservado-abierto), 
factor B (inteligencia baja-alta), factor C (afectado por los sentimientos-emocionalmente estable), factor D (calmoso-excitable), factor $E$ (sumiso-dominante), factor $F$ (sobrio-entusiasta), factor G (despreocupado-consciente), factor $\mathrm{H}$ (cohibido-emprendedor), factor I (sensibilidad blanda-sensibilidad dura), factor J (seguro-dubitativo), factor N (sencillo-astuto), factor O (sereno-aprensivo) y factor Q4 (relajado-tenso). También mide 2 de segundo orden: QI (ajuste-ansiedad) y QII (introversión-extroversión) ${ }^{16}$.

La aplicación se realizó a la población objeto de estudio en forma colectiva en dos tiempos, de acuerdo con recomendaciones del manual de la prueba. Teniendo en cuenta el propósito de la investigación, se escogieron 8 de los 13 factores de primer orden (A, D, E, F, H, J, O, Q4), en los cuales se consideró que podría identificarse la influencia de los ER en los rasgos de personalidad, teniendo en cuenta las teorías de personalidad frente a los patrones de comportamiento que están determinados por la influencia del ambiente en la interacción diaria de las personas ${ }^{16}$.

\section{Análisis estadístico}

En el presente estudio se descartó el manejo de datos de miopía por presentarse un único caso; por lo anterior, todas las variables se analizaron teniendo en cuenta el diagnóstico de emetropía, hipermetropía y astigmatismo. Se usaron frecuencias simples y relativas, media (M) y DE para la presentación de los datos descriptivos. La asociación entre los patrones de crecimiento, hábitos nutricionales y ER se realizó a través de la prueba de varianza ANOVA. Los resultados de estilos de vida fueron expresados por medio de estadísticos descriptivos y resumidos gráficamente. Para establecer la asociación entre los rasgos de personalidad y los ER se ejecutó la prueba de varianza ANOVA a partir de los resultados de cada decatipo por escala y factores de segundo orden. Los decatipos se distribuyen en una escala de 10 puntos equidistantes en unidades típicas, con una media de 5.5 y una DE de 2 decatipos. Siendo así, los valores entre 5-6 fueron considerados dentro del promedio de media de la población, y los valores entre 2-3 y 8-9 fueron considerados con gran desviación, mientras los valores de 1 y 10 fueron considerados como desviaciones extremas.

Finalmente, se categorizaron las variables relacionadas con el diagnóstico nutricional, los estilos de vida y los rasgos de personalidad para establecer asociación con los ER por medio de la prueba Chi cuadrado de Pearson, expresando los datos de acuerdo con el resultado de significancia $\mathrm{p}$. El nivel de significancia establecido para este estudio fue de $p<0.05$. El análisis estadístico se llevó a cabo con el software SPSS 25.

\section{Consideraciones éticas}

Este estudio se adhiere a lo establecido para investigación en seres humanos de acuerdo con la Asociación Médica Mundial y la Declaración de Helsinki; y por la Resolución 8430 de 1993 del Ministerio de Salud de la República de Colombia, que establece las consideraciones relacionadas con investigación de seres humanos y en población menor de edad. El estudio fue aprobado por el Comité de Ética de la Universidad Antonio Nariño de la ciudad de Bogotá, Colombia, bajo el número de protocolo 2017226. Así mismo, fue presentado y aprobado por las directivas de un colegio ubicado en la localidad de Ciudad Bolívar en Bogotá. Los procedimientos de recolección de datos se llevaron a cabo previo consentimiento informado de padres y cuidadores y asentimiento informado por parte de los niños con competencias de lecto-escritura.

\section{Resultados}

La población fue evaluada el segundo semestre de 2018. En el estudio participaron 64 niñas y 85 niños, sin exclusión por origen étnico; en su mayor parte pertenecientes al grado segundo de primaria. En cuanto a las características sociodemográficas, destacan las condiciones socioeconómicas de la población por bajos niveles de ingresos económicos y estratificación social; así mismo, el nivel educativo de padres y cuidadores en la mayor proporción no supera la formación secundaria.

En cuanto al diagnóstico de ER se halló una prevalencia del $33.5 \%$ de hipermetropía y del $18.7 \%$ de astigmatismo, con mayor frecuencia de hipermetropía en niños y de astigmatismo en niñas. Por otra parte, se observaron diferencias significativas entre el diagnóstico de ER y el curso de formación $(p<0.05)$, con base en mayor frecuencia de astigmatismo al incrementarse el grado de formación, y comportamiento inverso en relación con la hipermetropía; subsecuentemente, este comportamiento es el mismo en relación con la edad.

Datos relacionados con aspectos sociales, como ingresos familiares, estrato social o educación familiar, no mostraron diferencias estadísticamente significativas, pero sí mostraron mayor frecuencia de hipermetropía en 
Tabla 1. Características sociodemográficas de la población y errores de refracción (Bogotá, Colombia; $n=149$ )

\begin{tabular}{|c|c|c|c|c|c|c|c|}
\hline & Emetropía & Hipermetropía & Astigmatismo & & Emetropía & Hipermetropía & Astigmatismo \\
\hline & n (\%) & n (\%) & $n(\%)$ & & $n(\%)$ & n $(\%)$ & n $(\%)$ \\
\hline Sexo & & & & Estrato social & & & \\
\hline Mujer & $29(40.8)$ & $20(40.0)$ & $15(53.6)$ & Bajo-bajo & $30(42.3)$ & $26(52.0)$ & $16(57.1)$ \\
\hline Hombre & $42(59.2)$ & $30(60.0)$ & $13(46.4)$ & Bajo & $40(56.3)$ & $24(48.0)$ & 12 (42.9) \\
\hline Curso & & & & Medio-bajo & $1(1.4)$ & 0 & 0 \\
\hline Primero & $11(15.5)$ & $29(58.0)$ & $7(25.0)$ & Nivel educativo madre & & & \\
\hline Segundo & $31(43.7)$ & $20(40.0)$ & $10(35.7)$ & Ninguno & $1(1.4)$ & 0 & 0 \\
\hline Tercero & $29(40.8)$ & $1(2.0)$ & $11(39.3)$ & Primaria & $7(9.9)$ & $5(10.0)$ & $4(14.3)$ \\
\hline Edad (años) & & & & Secundaria & $42(59.2)$ & $32(64.0)$ & $13(46.4)$ \\
\hline 6 & $3(4.2)$ & $15(30.0)$ & $2(7.1)$ & Técnico & $16(22.5)$ & $11(22.0)$ & $7(25.0)$ \\
\hline 7 & $25(35.2)$ & $24(48.0)$ & $10(35.7)$ & Universitario & $5(7.0)$ & $2(4.0)$ & $4(14.3)$ \\
\hline 8 & $42(59.2)$ & $11(22.0)$ & $15(53.6)$ & Nivel educativo padre & & & \\
\hline 9 & $1(1.4)$ & 0 & $1(3.6)$ & Ninguno & $4(5.6)$ & $1(2.0)$ & 0 \\
\hline Ingresos familiares & & & & Primaria & $7(9.9)$ & $12(24.0)$ & $3(10.7)$ \\
\hline Menos de 1 SMMLV* & $7(9.9)$ & $12(24.0)$ & $5(17.9)$ & Secundaria & $50(70.4)$ & $30(60.0)$ & $18(64.3)$ \\
\hline Entre 1 y 2 SMMLV & 55 (77.5) & $27(54.0)$ & $20(71.4)$ & Técnico & $5(7.0)$ & $4(8.0)$ & $5(17.9)$ \\
\hline Entre 2 y 3 SMMLV & $7(9.9)$ & $8(16.0)$ & $2(7.1)$ & Tecnólogo & 0 & $2(4.0)$ & 0 \\
\hline Más de 3 SMMLV & $2(2.8)$ & $3(6.0)$ & $1(3.6)$ & Universitario & $5(7.0)$ & $1(2.0)$ & $2(7.1)$ \\
\hline
\end{tabular}

condiciones socioeconómicas más bajas en comparación a niños emétropes y con astigmatismo (Tabla 1).

En relación con los patrones de crecimiento y hábitos nutricionales, se observó mayor peso y estatura en niños emétropes $(M=26.58 \pm 5.96 ; 1.25 \pm 0.06)$ frente a niños y niñas con hipermetropía ( $M=24.30 \pm 4.84$; $1.21 \pm 0.07)$ y astigmatismo $(M=25.02 \pm 3.79$; $1.24 \pm 0.06$ ), y se hallaron diferencias estadísticamente significativas con el parámetro de talla como indicador del estado nutricional acumulativo $(p=0.046)$. Sin embargo, los resultados frente al indicador global de sobrepeso o delgadez, dado por el IMC, no mostraron diferencias estadísticamente significativas entre niños comparados por ER.

Los hábitos de consumo de alimentos saludables como verduras y frutas, en general, no superaban dos porciones diarias, a excepción del consumo de lácteos, en el cual se identificó mayor consumo por parte de los niños y niñas con hipermetropía, y fue estadísticamente significativo (Tabla 2). Por otra parte, el consumo de alimentos no saludables, como productos empacados y comidas rápidas, es bajo, y se limita a menos de un consumo a la semana en el caso de comidas rápidas y una porción de productos empacados al día. Aun así, se identificaron diferencias estadísticamente significativas en el consumo de bebidas azucaradas entre niños con ER y niños emétropes, que fue menor en estos últimos.

Por otra parte, el estudio arrojó diferencias estadísticamente significativas entre el promedio de sueño de los niños comparados por ER, y el tiempo de sueño fue mayor en niños con hipermetropía frente a los otros dos grupos de estudio (Tabla 2).

El estilo de vida de los niños y niñas participantes en el estudio, en general, mostró un comportamiento positivo en la mayoría de las dimensiones analizadas. Las habilidades de comunicación y expresión de cariño por parte de los niños mostraron resultados por encima del $50.7 \%$, y la recepción de cariño por parte de padres y cuidadores fue percibido con rangos mayores al $72 \%$ de la población, lo que arrojó una puntuación global alta. 
Tabla 2. Errores de refracción y patrones de crecimiento, hábitos nutricionales, sueño y lectura

\begin{tabular}{|c|c|c|c|c|c|c|c|c|c|c|}
\hline & \multicolumn{3}{|c|}{ Emetropía (n = 71) } & \multicolumn{3}{|c|}{ Hipermetropía (n = 50) } & \multicolumn{3}{|c|}{ Astigmatismo (n = 28) } & \multirow{3}{*}{$\begin{array}{c}\text { Valor } \\
p^{*}\end{array}$} \\
\hline & \multirow[t]{2}{*}{ M/DE } & \multicolumn{2}{|c|}{ IC $95 \%$} & \multirow[t]{2}{*}{ M/DE } & \multicolumn{2}{|c|}{ IC 95\% } & \multirow[t]{2}{*}{ M/DE } & \multicolumn{2}{|c|}{ IC 95\% } & \\
\hline & & $\begin{array}{c}\text { Límite } \\
\text { inferior }\end{array}$ & $\begin{array}{l}\text { Límite } \\
\text { superior }\end{array}$ & & $\begin{array}{l}\text { Límite } \\
\text { inferior }\end{array}$ & $\begin{array}{l}\text { Límite } \\
\text { superior }\end{array}$ & & $\begin{array}{l}\text { Límite } \\
\text { inferior }\end{array}$ & $\begin{array}{l}\text { Límite } \\
\text { superior }\end{array}$ & \\
\hline Peso (kg) & $26.58 \pm 5.96$ & 25.176 & 28.001 & $24.3 \pm 4.84$ & 22.924 & 25.676 & $25.02 \pm 3.79$ & 23.549 & 26.494 & 0.056 \\
\hline Talla (m) & $1.25 \pm 0.06$ & 1.240 & 1.272 & $1.21 \pm 0.07$ & 1.197 & 1.238 & $1.24 \pm 0.06$ & 1.221 & 1.271 & 0.009 \\
\hline IMC & $16.71 \pm 2.67$ & 16.080 & 17.347 & $16.28 \pm 2.17$ & 15.666 & 16.900 & $16.06 \pm 1.70$ & 15.407 & 16.732 & 0.396 \\
\hline Porciones fruta/día & $1.61 \pm 0.93$ & 1.399 & 1.840 & $1.62 \pm 1.10$ & 1.306 & 1.934 & $1.64 \pm 1.47$ & 1.072 & 2.213 & 0.995 \\
\hline Porciones verdura/día & $1.12 \pm 0.84$ & 0.927 & 1.327 & $1.28 \pm 0.90$ & 1.023 & 1.537 & $1.32 \pm 1.18$ & 0.861 & 1.782 & 0.541 \\
\hline $\begin{array}{l}\text { Bebidas azucaradas/ } \\
\text { día }\end{array}$ & $0.88 \pm 0.85$ & 0.685 & 1.090 & $1.56 \pm 1.97$ & 1.000 & 2.120 & $1 \pm 1.24$ & 0.516 & 1.484 & 0.030 \\
\hline Lácteos/día & $1.94 \pm 1.18$ & 1.664 & 2.223 & $2.48 \pm 1.44$ & 2.069 & 2.891 & $1.71 \pm 1.01$ & 1.321 & 2.107 & 0.017 \\
\hline $\begin{array}{l}\text { Alimentos } \\
\text { empacados/día }\end{array}$ & $1.18 \pm 1.00$ & 0.945 & 1.421 & $1.04 \pm 0.94$ & 0.771 & 1.309 & $1.25 \pm 0.96$ & 0.875 & 1.625 & 0.606 \\
\hline $\begin{array}{l}\text { Comidas rápidas/ } \\
\text { semana }\end{array}$ & $0.59 \pm 0.70$ & 0.424 & 0.759 & $0.72 \pm 0.92$ & 0.457 & 0.983 & $0.42 \pm 0.50$ & 0.233 & 0.624 & 0.265 \\
\hline Horas de sueño/día & $8.63 \pm 0.97$ & 8.403 & 8.864 & $9.04 \pm 1.08$ & 8.731 & 9.349 & $8.46 \pm 1.03$ & 8.063 & 8.866 & 0.032 \\
\hline $\begin{array}{l}\text { Frente al PC o TV } \\
\text { entre semana }^{\dagger}\end{array}$ & $2.81 \pm 3.15$ & 2.070 & 3.564 & $3.88 \pm 2.25$ & 3.239 & 4.521 & $2.5 \pm 2.78$ & 1.421 & 3.579 & 0.057 \\
\hline $\begin{array}{l}\text { Frente al PC o TV } \\
\text { fines de semana }\end{array}$ & $3.35 \pm 2.71$ & 2.711 & 3.994 & $3.6 \pm 3.30$ & 2.660 & 4.540 & $2.75 \pm 3.05$ & 1.567 & 3.933 & 0.482 \\
\hline $\begin{array}{l}\text { Leer o dibujar entre } \\
\text { semana }^{\dagger}\end{array}$ & $2.09 \pm 2.06$ & 1.610 & 2.587 & $2.56 \pm 2.74$ & 1.781 & 3.339 & $2.25 \pm 1.77$ & 1.561 & 2.939 & 0.545 \\
\hline $\begin{array}{l}\text { Leer o dibujar fines de } \\
\text { semana }^{\dagger}\end{array}$ & $1.90 \pm 1.63$ & 1.515 & 2.288 & $2.52 \pm 1.84$ & 1.996 & 3.044 & $1.92 \pm 1.80$ & 1.229 & 2.628 & 0.132 \\
\hline
\end{tabular}

En cuanto a la alimentación, los niños informaron acceso a alimentación variada y saludable por encima del $40 \%$, y consumo de desayuno, almuerzo y comida en el $88 \%$ de los casos. Por otra parte, el consumo de comidas poco saludables estaba restringida a una periodicidad semanal o quincenal en el $76.1 \%$ de la población.

La exposición a tóxicos y alcohol fue limitada, y la tendencia arrojó respuesta positiva; sin embargo, se relacionó como un factor de riesgo en la población de estudio. Se identificó que el $14.1 \%$ de niños emétropes había fumado y el $5.6 \%$ había consumido alcohol alguna vez. Lo anterior puede relacionarse con la convivencia con personas de consumo constante de alcohol o cigarrillo, el cual fue en niños emétropes del $11.3 \%$ en comparación con niños hipermétropes y con astigmatismo (2 y 10.7\%, respectivamente).
Los hábitos de sueño, en general, mostraron que la percepción de descanso para todos los niños está por encima del $62 \%$, y las dimensiones relacionadas con tipo de personalidad e imagen interior mostraron adecuado manejo de situaciones estresantes, capacidad de relajación, estabilidad emocional y buen humor. Aun así, se logró determinar que los niños con hipermetropía tienen mayor descontento con el estudio (51.4\%) en comparación con los niños emétropes (37.8\%) y con astigmatismo (10.8\%), lo que arroja diferencias estadísticamente significantes $(p=0.046)$. Emociones como el estrés fueron mínimamente percibidas, pero los sentimientos de tristeza fueron percibidos en un mayor porcentaje en niños con hipermetropía (66.7\%) en comparación con los dos grupos de comparación (emétropes $22.2 \%$, astigmáticos $11.1 \%$ ). 


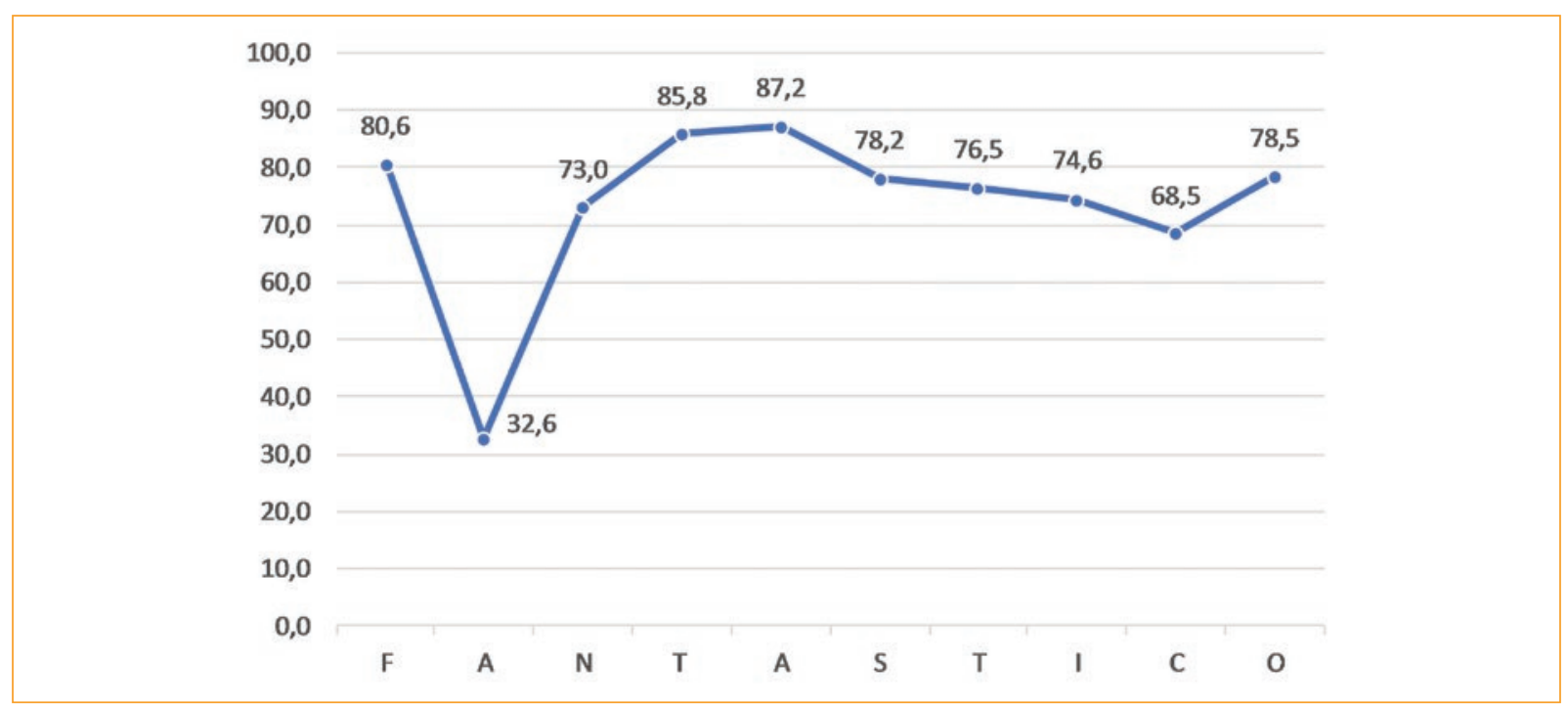

Figura 1. Tendencia por dimensiones de estilos de vida (Bogotá, Colombia; $n=149$ ). Se muestran las tendencias de cada una de las escalas del instrumento FANTÁSTICO, el cual evalúa el estilo de vida en población infantil. Los datos muestran la tendencia por cada una de las escalas con base en estadística descriptiva (porcentajes). F: familia y amigos; A: actividad física y social; N: nutrición; T: tóxicos; A: alcohol; S: sueño y estrés; T: tipo de personalidad; I: imagen interior; $\mathrm{C}$ : control de la salud y sexualidad; 0: orden

Finalmente, se identificó una tendencia irregular en el control médico y cuidado de la sexualidad en todos los niños y niñas, con una insatisfacción con la percepción de la apariencia física en el $52 \%$ de niños con hipermetropía, porcentaje mayor al resto, lo que se refleja en la tendencia global de la dimensión. Se destaca limitada participación en actividades sociales y recreativas teniendo en cuenta que el $57.1 \%$ de los niños y niñas nunca participaban en actividades juveniles o hacían ejercicio físico programado. La figura 1 muestra las tendencias globales de las dimensiones evaluadas en el estilo de vida, y refleja principalmente el impacto negativo en el resultado global de las actividades sociales y ejercicio físico, y el cuidado de la salud en el estilo de vida de niños y niñas (Fig. 1).

Los resultados de los rasgos de personalidad de los niños y niñas participantes permitieron evidenciar habilidades promedio para el control de la ansiedad, factor QI, Io que refleja estabilidad de la personalidad. Las escalas que conforman este factor mostraron igual tendencia frente a la media de la población en las escalas D, F, H, O y Q4, lo que refleja niños normorreactivos a estímulos, provenientes de un entorno familiar relativamente seguro, con respuesta emocional positiva hacia las personas, expresivos y activos, pocos aprensivos y con conductas positivas de sociabilidad. Sin embargo, se identificaron diferencias estadísticamente significativas en la escala $\mathrm{J}$ entre niños con astigmatismo y niños con hipermetropía, estos últimos eran según los criterios de la escala más individualistas, motrizmente reprimidos y despreciativos con los demás en comparación con niños con $\mathrm{J}$ - .

Por su parte, el factor QII de introversión-extraversión está representado por las escalas A, E, F Y Q4, que también mostraron resultados promedios frente a la media poblacional y reflejaron niños adaptados al medio escolar, con conductas positivas, constructivas y adecuado ambiente familiar (Tabla 3 ).

Finalmente, la tabla 4 nos muestra los resultados frente al análisis de asociación entre variables categorizadas y los ER. Se encontró la mayor parte de niños y niñas con adecuado estado nutricional y con buenos estilos de vida en niños emétropes y con ER. En cuanto a los rasgos de personalidad, se evidenció en su mayor parte niños y niñas con características promedio, que permiten su adaptabilidad al ambiente escolar, sin diferencias estadísticamente significativas (Tabla 4).

\section{Discusión}

Un gran número de estudios que identifican los factores de riesgo asociados a la miopía en la población infantil se han llevado a cabo, sin embargo, en relación con la hipermetropía y el astigmatismo, los estudios han sido mucho menores. Lo anterior puede considerarse a partir del comportamiento epidemiológico de 
Tabla 3. Asociación entre perfil psicológico y errores de refracción

\begin{tabular}{|c|c|c|c|c|c|c|c|}
\hline & \multirow{2}{*}{\multicolumn{2}{|c|}{$\begin{array}{l}\text { Emetropía } \\
\qquad(n=71)\end{array}$}} & \multirow{2}{*}{\multicolumn{2}{|c|}{$\begin{array}{l}\text { Hipermetropia } \\
\qquad(\mathrm{n}=50)\end{array}$}} & \multirow{2}{*}{\multicolumn{2}{|c|}{$\begin{array}{c}\text { Astigmatismo } \\
(n=28)\end{array}$}} & \multirow[t]{3}{*}{ Valor $p^{*}$} \\
\hline & & & & & & & \\
\hline & M/DE & IC $95 \%$ & M/DE & IC $95 \%$ & M/DE & IC $95 \%$ & \\
\hline \multicolumn{8}{|c|}{ Escalas } \\
\hline A: reservado-abierto & $5.41 \pm 1.924$ & $(4.95-5,86)$ & $5.92 \pm 2.098$ & $(5.32-5.86)$ & $6.04 \pm 2.151$ & $(5.20-6.87)$ & 0.247 \\
\hline D: calmoso-excitable & $4.55 \pm 1.918$ & $(4.10-5.00)$ & $4.82 \pm 2.077$ & $(4.23-5.41)$ & $4.82 \pm 1.887$ & $(4.09-5.55)$ & 0.703 \\
\hline E: sumiso-dominante & $4.92 \pm 1.998$ & $(4.44-5.39)$ & $4.54 \pm 2.022$ & (3.97-5.11) & $5.11 \pm 1.685$ & $(4.45-5.76)$ & 0.408 \\
\hline F: sobrio-entusiasta & $5.54 \pm 1.747$ & (5.12-5.95) & $5.30 \pm 1.619$ & $(4.84-5.76)$ & $6.00 \pm 2.091$ & $(5.19-6.81)$ & 0.251 \\
\hline H: cohibido-emprendedor & $5.15 \pm 1.555$ & $(4.79-5.52)$ & $5.26 \pm 2.048$ & $(4.68-5.84)$ & $5.79 \pm 1.424$ & $(5.23-6.34)$ & 0.253 \\
\hline J: seguro-dubitativo & $5.59 \pm 1.745$ & $(5.18-6.00)$ & $6.22 \pm 1.753$ & $(5.72-6.72)$ & $5.04 \pm 1.915$ & $(4.29-5.78)$ & 0.017 \\
\hline 0: sereno-aprensivo & $5.99 \pm 1.840$ & $(5.53-6.42)$ & $5.86 \pm 2.129$ & $(5.26-6.46)$ & $5.86 \pm 1.779$ & $(5.17-6.55)$ & 0.923 \\
\hline Q4: relajado-tenso & $5.39 \pm 1.938$ & (4.94-5.85) & $5.24 \pm 1.901$ & $(4.70-5.78)$ & $4.64 \pm 1.850$ & $(3.93-5.36)$ & 0.212 \\
\hline \multicolumn{8}{|c|}{ Factores de segundo orden } \\
\hline Ol: ajuste-ansiedad & $5.94 \pm 1.851$ & $(5.51-6.38)$ & $6.20 \pm 1.972$ & $(5.65-6.75)$ & $5.50 \pm 1.528$ & (4.91-6.09) & 0.269 \\
\hline Qll: introversión-extraversión & $6.52 \pm 1.847$ & $(5.18-6.06)$ & $5.96 \pm 1.795$ & $(5.45-6.47)$ & $5.71 \pm 1.560$ & $(5.11-6.32)$ & 0.582 \\
\hline
\end{tabular}

Tabla 4. Asociación de variables de estudio con errores de refracción (Bogotá, Colombia; $n=149$ )

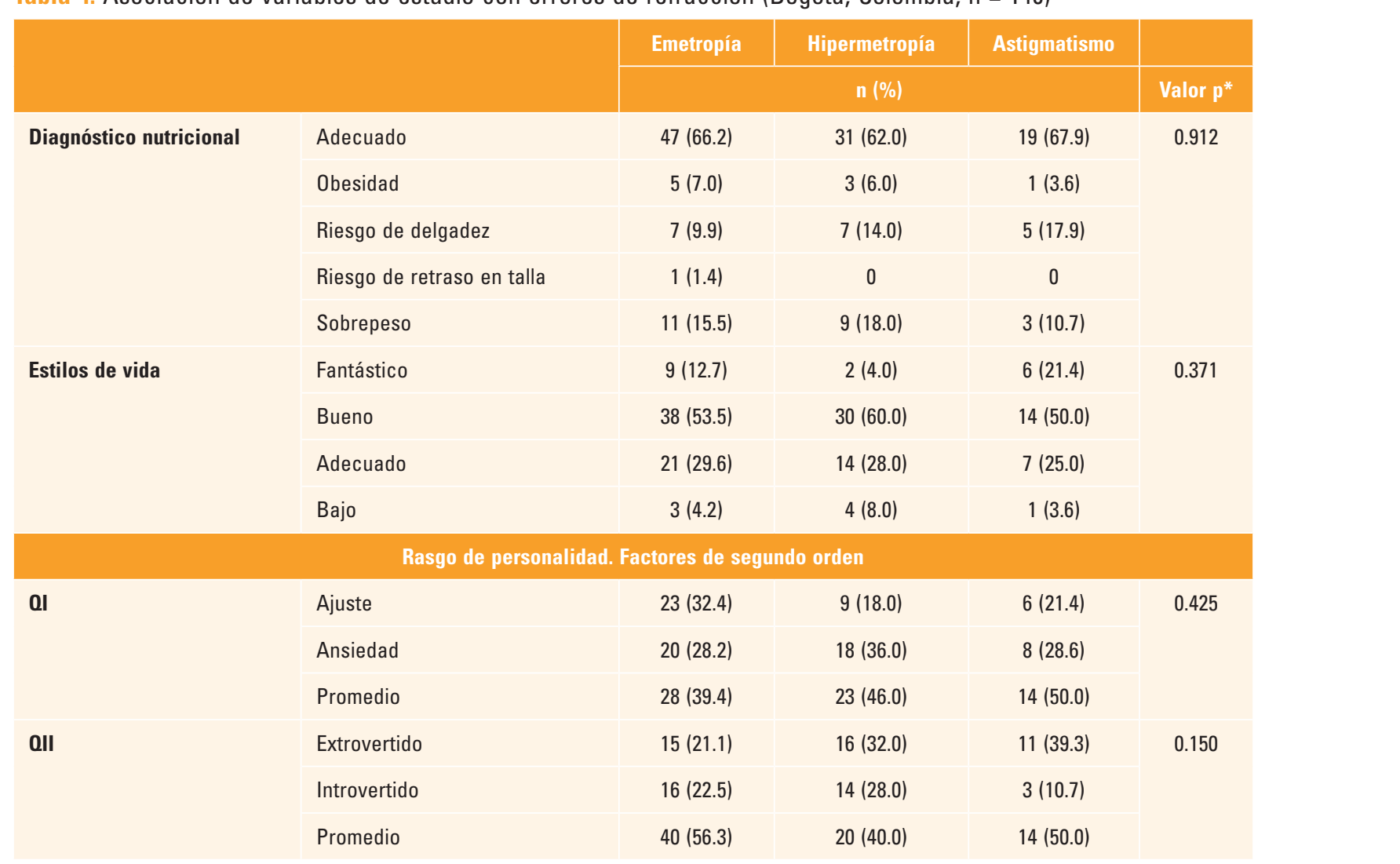

${ }^{*}$ Chi cuadrado de Pearson. 
los ER, ya que, en regiones de mayor publicación, la prevalencia de miopía es mayor que en la región de las Américas, lo cual inclusive en nuestro estudio determinó la exclusión de un único caso hallado.

En este sentido, la prevalencia de ER estimada a nivel mundial en niños con miopía, hipermetropía y astigmatismo es del $11.7,4.6$ y $14.9 \%$, respectivamente, y específicamente la prevalencia de hipermetropía y astigmatismo es mayor en la región de las Américas en comparación con la prevalencia global (14.3\% y 27.2\%)1. En Colombia, reportes de prevalencia con utilización de refracción ciclopégica no han sido reportados, no obstante, el Instituto Nacional de Salud, en el año 2000, informó en población de 5 a 14 años prevalencias de hipermetropía del $59.2 \%$, de astigmatismo del $28.2 \%$, de miopía del $4.0 \%$ y de emetropía del $9.0 \%{ }^{17}$.

Nosotros encontramos que la prevalencia de hipermetropía fue mayor en comparación con la de astigmatismo, lo que difiere de la tendencia en los continentes europeo y asiático y concuerda con la región americana, en donde estos ER son identificados como las alteraciones visuales con mayor frecuencia en la población infantil asociadas a posibles factores genéticos y ambientales propios de la región. Aun así, también identificamos disminución en la frecuencia de hipermetropía con el incremento de la edad, manteniendo la tendencia hacia la emetropización, por los cambios en la curvatura corneal, la longitud axial y la potencia del cristalino expresados a partir de los 6 años $^{18}$, lo cual permite deducir un adecuado proceso de emetropización en nuestra población. En relación con el astigmatismo, aunque la prevalencia es menor y similar con otros resultados ${ }^{19}$, su tendencia a aumentar con la edad es significativa en nuestra población, por lo tanto, identificar el astigmatismo en la población pediátrica es primordial por el riesgo de desarrollar ambliopía, especialmente en astigmatismos altos, y por la asociación reportada por la literatura entre el astigmatismo y el desarrollo de miopía ${ }^{20}$. Por otra parte, aunque las causas de astigmatismo no son claras, factores genéticos, presión sobre los parpados, tensión de los músculos extraoculares, edad gestacional y bajo peso al nacer son condiciones que tienen un papel en el desarrollo de astigmatismo, y deben tenerse en cuenta, así como fallas en la emetropización y factores socioambientales ${ }^{18}$.

La salud visual de nuestra población se caracterizó por una mayor prevalencia de hipermetropía en niños y astigmatismo en niñas, con un incremento del astigmatismo en la población general y disminución de la hipermetropía a mayor edad. Si bien no se encontró asociación entre el género con los ER, al igual que en el estudio de $\mathrm{Pi}$, las investigaciones relacionadas han encontrado mayor probabilidad de hipermetropía en niñas ${ }^{21-24}$, lo que difiere con nuestros resultados, por lo cual se hace necesario en estudios posteriores enfatizar en las diferencias comportamentales por sexo, en cuanto a factores socioambientales, culturales y hábitos de estudio.

En cuanto a los aspectos sociodemográficos, se encontró mayor prevalencia de ametropías en condiciones socialmente desfavorables. Tarczy-Hornoch, et al., identificaron una asociación de la falta de seguridad en salud y la menor educación familiar con una disminución de la visión ${ }^{25}$. En nuestro estudio, no pueden observarse resultados concluyentes frente a esas variables específicas por la homogeneidad social de la población, sin embargo, sí se logra contextualizar las diferencias globales entre niños emétropes y con ER a partir del análisis de aspectos nutricionales y estilos de vida como parámetros indirectos de la situación social de nuestros niños.

Los resultados frente al estado nutricional mostraron diferencias estadísticamente significativas entre niños y niñas con emetropías y ER en relación con la talla, sin ser claramente definido si la condición nutricional es un factor diferenciador, teniendo en cuenta los parámetros globales del estado nutricional. La literatura ha reportado la relación entre desnutrición y alteraciones visuales, sin embargo, la relación de la talla con los ER aún no está claramente documentada. Cabe resaltar que, en el presente estudio, los niños hipermétropes y astigmáticos presentaron una talla inferior a los niños con emetropía; aspecto que debe ser evaluado con mayor profundidad a partir de modelos ajustados de análisis.

En cuanto a los hábitos nutricionales y estilos de vida, el limitado consumo de frutas y verduras y las diferencias de consumo de lácteos entre niños con ametropías fue significativo, al igual que las diferencias entre el consumo de azúcar entre niños amétropes y emétropes. En general, los niños emétropes consumen menos porciones de azúcar que los niños con ametropía, especialmente hipermétropes. La literatura sugiere que las dietas altas en carbohidratos pueden causar cambios permanentes en el desarrollo y progresión de los ER, particularmente durante periodos de desarrollo infantil, por lo cual debe analizarse con mayor profundidad. De acuerdo a You, no hay evidencia que relacione el consumo de frutas y verduras con los $\mathrm{ER}^{26}$. Aun así, cabe destacar que nuestra población de estudio pertenece a zonas clasificadas por índices de 
pobreza que tienen acceso a servicios comunitarios de alimentación. En este sentido, es probable que su alimentación dentro de la jornada escolar incluya alimentos enriquecidos con vitamina $D$ y lácteos indispensables para el metabolismo del calcio y fosfato, lo cual determina la posterior actividad dopaminérgica en la retina, la esclera y crecimiento del globo ocular27-29. Además, el acceso limitado a comida rápida por cuenta de las condiciones económicas de la población se transforma en un factor protector, en gran medida por la conservación de un estado nutricional adecuado relacionado con el bajo consumo de grasa animal y carbohidratos simples, reportados por Terasaki, et al. como alimentos desencadenantes de hipercolesterolemia e insulinemia asociados con la longitud axial y la subsecuente elongación de las fibrillas de colágeno en la esclera ${ }^{30}$.

En relación con los hábitos de descanso y sueño, nosotros identificamos diferencias estadísticamente significativas del promedio de horas de sueño en niños con ametropías. Los niños astigmáticos en comparación con niños hipermétropes tenían menos horas de descanso. Al respecto, la literatura ha reportado que niños con menos de 7 horas de sueño por día tienen un riesgo 3.37 veces mayor de miopía que aquellos con más de 9 horas de sueño ${ }^{31,32}$; sin embargo, Sensaki, et al. concluyeron que no existe relación entre las horas de sueño y los ER en niños de 3 años ${ }^{26,33}$.

Por otra parte, los hábitos de exposición a pantallas de televisor y trabajo cercano relacionado con la lectura y dibujo arrojaron mayor tiempo en niños hipermétropes. Los resultados frente al impacto de estos hábitos en población infantil aún no son claros. En estudios con niños en Japón, los análisis no fueron significativamente diferentes comparando grupos con y sin ER; sin embargo, datos de progresión hacia la miopía han sido reportados en estudios españoles de población joven ${ }^{34}$.

En cuanto a la exposición a tóxicos, nosotros identificamos alta frecuencia e inicio temprano de consumo de alcohol y tabaco. La preocupación frente a la vulnerabilidad de la población debe ser motivo de intervención, pero en relación con los ER no hay una asociación significativa en este curso de vida, pero sí está claramente relacionada la exposición prenatal y posterior desarrollo de ambliopía ${ }^{25,34}$.

Las actividades físicas y de recreación en general fueron limitadas en nuestra población. Lo anterior puede considerarse teniendo en cuenta las limitadas posibilidades de acceso de nuestros niños a actividades deportivas por la condición socioeconómica e infraestructura recreativa del área de influencia del estudio, lo cual implica menor participación en actividades al aire libre y, por ende, sedentarismo. Al respecto, aún no es clara la relación entre la actividad física y los ER, sin embargo, Huang, et al. han reportado mayor prevalencia de entidades como la miopía por la menor actividad al aire libre $^{34}$, sin ser claro en el caso de la hipermetropía o el astigmatismo. En este sentido, el interés para el control de las repercusiones de los ER y la ausencia de actividad física debe enfocarse en el análisis de la comorbilidad de enfermedades no trasmisibles con la pérdida visual en cursos posteriores de la vida.

Los resultados frente a ER y rasgos de personalidad arrojaron estabilidad emocional en el mayor porcentaje de la población, caracterizando niños con adecuada respuesta emocional, adaptados al medio escolar, con conductas positivas y constructivas. Aun así, se encontraron diferencias estadísticamente significativas en rangos de personalidad relacionados con el control de la ansiedad entre niños con astigmatismo e hipermetropía, y estos últimos fueron de especial interés a partir de nuestros hallazgos por sus rasgos más individualistas, motrizmente reprimidos y despreciativos con los demás en comparación con niños con J-, en la escala duda-seguridad, lo que también ha sido reportado por Akcay, et al. en personas adultas ${ }^{5}$. De acuerdo con este autor, personas con ER son menos compasivos y más egocéntricos, y específicamente niños hipermétropes pueden tener características débiles frente a la empatía ${ }^{5}$, especialmente por el desarrollo de una personalidad exigente. Uretme, et al. relacionan estos tipos de personalidad con procesos de acomodación para borrar imágenes y posterior desarrollo de esotropía, y los comparan con niños que desarrollan ambliopía ametrópica, quienes conservan rasgos exigentes, perfeccionistas y prescriptivos, también presentes en niños con hipermetropía ${ }^{35}$. Por el contrario, niños con astigmatismo demuestran características más empáticas.

\section{Conclusión}

El análisis global de las variables en nuestra muestra no mostró diferencias estadísticamente significativas entre ametropías y emetropías en relación con los factores examinados: nutrición, actividad física y rasgos de personalidad. Sin embargo, este estudio puede considerarse un punto de partida para identificar la prevalencia y analizar las condiciones predisponentes de ER, especialmente en niños hipermétropes, en los cuales observamos resultados particulares en relación con estilos de vida, rasgos de personalidad y estabilidad emocional. En nuestra población de estudio, el 
estigmatismo tiende a aumentar con la edad, por lo que identificar precozmente este ER en la población infantil es primordial para disminuir el riesgo de desarrollar ambliopía.

\section{Limitaciones}

Nuestro estudio tuvo las siguientes limitaciones. Primero, probablemente el tamaño de la muestra no permitió encontrar asociación entre las múltiples variables analizadas. Segundo, las variables que analizan los aspectos nutricionales y psicológicos son muy amplias, por lo que se deben delimitar a futuro para su respectivo análisis. Tercero, no se hizo uso del autorrefractómetro para la toma de refracción, la cual solo se realizó por medio de retinoscopia. Cuarto, al ser un estudio transversal no se logró determinar la edad de inicio de anisometropía o el tipo de esotropía, las cuales pueden asociarse a la población latinoamericana. Quinto, el análisis de los factores de riesgo debe ser abordado a través de estudios longitudinales para identificar la condición de riesgo y la aparición del ER en etapas tempranas.

\section{Conflicto de intereses}

Los autores declaran que no existe conflicto de intereses.

\section{Financiación}

La presente investigación fue financiada por la Universidad Antonio Nariño de la ciudad de Bogotá, Colombia, bajo el número de protocolo 2017226.

\section{Responsabilidades éticas}

Este estudio se adhiere a lo establecido para investigación en seres humanos de acuerdo con la Asociación Médica Mundial y la Declaración de Helsinki, y por la Resolución 8430 de 1993 del Ministerio de Salud de la República de Colombia que establece las consideraciones relacionadas con investigación de seres humanos y en población menor de edad. El estudio fue aprobado por el Comité de Ética de la Universidad Antonio Nariño de la ciudad de Bogotá, Colombia, bajo el número de protocolo 2017226. Así mismo, fue presentado y aprobado por las directivas de un colegio ubicado en la localidad de Ciudad Bolívar en Bogotá. Los procedimientos de recolección de datos se llevaron a cabo, previo consentimiento informado de padres y cuidadores y asentimiento informado por parte de los niños con competencias de lecto-escritura

Protección de personas y animales. Los autores declaran que los procedimientos llevados a cabo en la investigación se basaron en el cumplimiento de las normas de investigación en seres humanos de acuerdo con la Asociación Médica Mundial y la Declaración de Helsinki.

Confidencialidad. Los autores expresan que los datos de los participantes del estudio fueron tratados bajo protocolo de confidencialidad y sigilo, de acuerdo con los protocolos institucionales.

Privacidad. Los autores han obtenido el consentimiento informado de padres y cuidadores de la población de estudio. Los documentos están bajo la custodia del autor de correspondencia.

\section{Bibliografía}

1. Hashemi H, Fotouhi A, Yekta A, Pakzad R, Ostadimoghaddam H, Khabazkhoob M. Global and regional estimates of prevalence of refractive errors: Systematic review and meta-analysis. J Curr Ophthalmol. 2018;30(1):3-22.

2. Rodruguez, I. Bartolomé FP, Ortiz MU, Villalobos PA, Rodríguez IU, Vallecilla MB, Cueto LF, et al. Estudio de la relación entre miopía y personalidad. Arch Soc Esp Oftalmol. 2015;90(8):365-72.

3. Hu H, Dai J, Chen M, Chen L, Jiang L, Lin R, et al. Effect of astigmatism on refraction in children with high hyperopia. Drug Discov Ther. 2017;10(6):323-8.

4. Van De Berg R, Dirani M, Chen CY, Haslam N, Baird PN. Myopia and personality: The genes in myopia (GEM) personality study. Investig Ophthalmol Vis Sci. 2008;49(3):882-6.

5. Akcay EK, Canan F, Simavli H, Dal D, Yalniz H, Ugurlu N, et al. Effect of refractive error on temperament and character properties. Int J Ophthalmol. 2015;8(1):72-6.

6. Loaiza DPB, Álvarez CV, Vargas LJ. Validación de contenido y adaptación del cuestionario Fantastico por técnica Delphi. Salud Uninorte. 2015;31(2):214-27.

7. Tideman JWL, Polling JR, Hofman A, Jaddoe VW, Mackenbach JP, Klaver CC, et al. Environmental factors explain socioeconomic prevalence differences in myopia in 6-year-old children. $\mathrm{Br} \mathrm{J}$ Ophthalmol. 2018;102(2):243-7.

8. Ip JM, Saw SM, Rose KA, Morgan IG, Kifley A, Wang JJ, et al. Role of near work in myopia: Findings in a sample of Australian school children. Investig Ophthalmol Vis Sci. 2008;49(7):2903-10

9. Organización Mundial de la Salud. Salud ocular universal. Un plan de acción mundial para 2014-2019. Madrid, España; 2014. [citado 5 de julio de 2019]. Disponible en: https://www.who.int/blindness/AP2014_19_Spanish.pdf?ua=1

10. Ministerio de Salud y Protección Social. Guía de Práctica Clínica para la prevención, la detección temprana, el diagnóstico, el tratamiento y el seguimiento de la ambliopía en menores de 18 años. Bogotá, Colombia 2016. [citado 3 de julio de 2019]. Disponible en: http://gpc.minsalud.gov. co/gpc_sites/Repositorio/Conv_637/GPC_d_refractivos/gpc_d_refractivos.aspx

11. Castagno VD, Fassa AG, Vilela MAP, Meucci RD, Resende DPM. Moderate hyperopia prevalence and associated factors among elementary school students. Cien Saude Colet [Internet]. 2015;20(5):1449-8.

12. Lizama Lefno A. Multidisciplinariedad en el Manejo de Enfermedades Crónicas. An la Fac Med. 2016;77(3):263-7.

13. World Health Organization. Assessment of the prevalence of visual impairment attributable to refractive error or other causes in school children. Protocol and Manual. Geneve. 2007. [citado 7 de julio de 2019] Disponible en: https://www.who.int/blindness/causes/RESCProtocol.pdf

14. Ministerio de Salud y Protección Social. Resolución 2465 de 2016. Bogotá, Colombia; 2016. [citado Julio 15 de 2019] Disponible en: https:// www.minsalud.gov.co/Normatividad_Nuevo/Forms/DispForm.aspx?ID=4909

15. Betancurth Loaiza DP, Vélez Álvarez C, Jurado Vargas L. Validation and adaptation of the Fantastico questionnaire by Delphi technique. Salud Uninorte. 2015 May;31(2):214-27. 
16. Coan RW, Cattell RB. Cuestionario de personalidad para niños. TEA Ediciones, $8^{\text {th }}$ ed. Madrid; 2013. $47 \mathrm{p}$.

17. Hernández Luna CP, Barrera Santos DC, Guiza Segura C, Rodríguez Malagón JP, Montaña Ludeman WE, et al. Estudio de prevalencia en salud visual en una población escolar de Bogotá, Colombia, 2000. Cienc Tecnol para la Salud Vis y Ocul. 2003;0(1):11-23.

18. Castro Piña S, Rey Rodríguez DV, Álvarez Peregrina C, Moreno Montoya J. Proceso de emetropización y desarrollo de miopía en escolares. Cienc Tecnol para la Salud Vis y Ocul. 2018;16(1):87-93.

19. Giordano L, Friedman DS, Repka MX, Katz J, Ibironke J, Hawes P, et al. Prevalence of Refractive Error among Preschool Children in an Urban Population: The Baltimore Pediatric Eye Disease Study. Ophthalmology. 2009;116(4):739-46.

20. Tong L, Saw SM, Carkeet A, Chan WY, Wu HM, Tan D. Prevalence rates and epidemiological risk factors for astigmatism in Singapore school children. Optom Vis Sci. 2002;79(9):606-13.

21. Guo X, Fu M, Ding X, Morgan IG, Zeng Y, He M. Significant Axial Elongation with Minimal Change in Refraction in 3- to 6-Year-Old Chinese Preschoolers: The Shenzhen Kindergarten Eye Study. Ophthalmology. 2017;124(12):1826-38

22. Guo $Y$, Liu LJ, Tang $P, \operatorname{Lv} Y Y$, Feng $Y, X u$ L, et al. Outdoor activity and myopia progression in 4-year follow-up of Chinese primary school children: The Beijing Children Eye Study. PLoS One. 2017; 12(4):1-14

23. Lau JK, Vincent SJ, Collins MJ, Cheung SW, Cho P. Ocular higher-orde aberrations and axial eye growth in young Hong Kong children. Sci Rep. 2018;8(1):2-11.

24. Pi LH, Chen L, Liu Q, Ke N, Fang J, Zhang S, et al. Refractive status and prevalence of refractive errors in suburban school-age children. Int J Med Sci. 2010;7(6):342-53.
25. Tarczy-Hornoch K, Varma R, Cotter SA, McKean-Cowdin R, Lin JH, Borchert MS, et al. Risk Factors for Astigmatism in Preschool Children. Ophthalmology. 2011;118(11)

26. You QS, Wu LJ, Duan JL, Luo YX, Liu LJ, Li X et al. Factors Associated with Myopia in School Children in China: The Beijing Childhood Eye Study. Baird P, editor. PLoS One. 2012 Dec 27;7(12)

27. Choi JA, Han K, Park Y-M, La TY. Low serum 25-hydroxyvitamin D is associated with myopia in Korean adolescents. Invest Ophthalmol Vis Sci. 2014 Apr;55(4):2041-7.

28. Ramamurthy D, Lin Chua SY, Saw S-M. A review of environmental risk factors for myopia during early life, childhood and adolescence. Clin Exp Optom. 2015 Nov;98(6):497-506.

29. Wilcox T, Hirshkowitz A. Boas D. The effect of color priming on infant brain and behavior. Neuroimage. 2015;85(0 1):1-27.

30. Terasaki H, Yamashita T, Yoshihara N, Kii Y, Sakamoto T. Association of lifestyle and body structure to ocular axial length in Japanese elementary school children. BMC Ophthalmol. 2017;17(1):1-7.

31. Jee D, Morgan IG, Kim EC. Inverse relationship between sleep duration and myopia. Acta Ophthalmol. 2016;94(3):e204-10.

32. Gong Y, Zhang X, Tian D, Wang D, Xiao G. Parental myopia, near work, hours of sleep and myopia in Chinese children. Health (Irvine Calif). 2014;06(01):64-70.

33. Sensaki S, Sabanayagam C, Chua S, Htoon HM, Broekman BFP, Thiam DGY, et al. Sleep Duration in Infants Was Not Associated With Myopia at 3 Years. Asia-Pacific J Ophthalmol. 2018;7(2):102-8.

34. Huang L, Kawasaki H, Yasuda R, Sakai R. Relationship between Visual Acuity and Lifestyle: A Cross-Sectional Study in Japanese Children. Hiroshima J Med Sci. 2018;67(4):1-2.

35. Uretmen O, Kesikci H, Erermis S, Kose S, Pamukcu K, Aydin C. Could personality traits modify the response to uncorrected high hyperopia? J AAPOS. 2005;9(5):480-4. 